\title{
VIII CONGRESO
}

Jmiversidad de Alicante, 9 y 10 de Jumnio/2016

TITULO: Validez de contenido de un cuestionario para medir la implicación de las enfermeras clínicas en la formación de estudiantes de Grado en Enfermería

Title: Content validity of a questionnaire to measure the involvement of clinical nurses in training students of Degree in Nursing

PALABRAS CLAVE: Enfermeria, educacion en enfermeria, tutoria, estudio de validación. Keywords: Nursing; Nursing education; Clinical Clerkship; Mentorship; Validation study

OBJETIVO:EL objetivo de este trabajo es presentar los resultados de la validez de contenido del proceso de desarrollo de un cuestionario para medir la implicación de las enfermeras clínicas en la formación de estudiantes de Grado en Enfermería.

METODOLOGIA: Para realizar la validez del contenido, se solicitó la colaboración de 5 personas expertas, siguiendo la metodología de Polit et al (2005), formando un panel de expertos en este área de conocimiento. Los criterios de selección de los expertos fueron: Doctor o Master en Enfermeria, mas de 10 años de experiencia docente con estudiantes de Enfermería en asignaturas con práctica clínica, procedencia de diversas universidades Españolas. A los expertos se les envió por correo electrónico la versión inicial del cuestionario, compuesto por 43 ítems, y se les solicitó que, valoraran la idoneidad y pertinencia de los ítems que componían cada dimensión evaluando mediante una escala Likert de 4 puntos. Los expertos dispusieron de 1 semana para completar la valoración. Se les indicó también la necesidad de plantear algún ítem de manera alternativa si no se comprendía suficientemente bien. Así mismo se les pidió que valorasen la adecuación global del cuestionario.

El cuestionario inicial que se envió a los expertos estaba formado por 4 dimensiones con un total de 43 ítems. El análisis de datos se realizó siguiendo la metodología de Polit y Beck (2006), calculando el índice de validez de contenido (I-CVI) y el coeficiente modificado de Kappa (K) para cada ítem del cuestionario. También se calculó el coeficiente global de validez de contenido (S-VCl) para cada dimensión y para el global del cuestionario. RESULTADOS: Tras el análisis, todas las dimensiones de cuestionario obtuvieron puntuaciones de S-CVI superiores a 0,8 , excepto una dimensión, que obtuvo una puntuación ligeramente inferior. Los ítems que obtuvieron un I-CVI elevado permanecieron en el cuestionario, otros fueron eliminados, con lo que se elaboró una nueva version del cuestionario para continuar con el proceso de validación. DISCUSIÓN: El aprendizaje clínico es un componente fundamental de la formación en enfermería y las enfermeras clínicas tienen un papel importante en la adquisición de competencias durante las prácticas clínicas de los estudiantes de enfermería. Se han realizado diversos estudios a través de cuestionarios para recoger aspectos relevantes relacionados con el aprendizaje clínico, desde la perspectiva del estudiantado, para observar cómo perciben la práctica clínica, pero no se han encontrado cuestionarios para evaluar la participación de las enfermeras clínicas desde su propia perspectiva.

CONCLUSIÓN: La validez de contenido de este cuestionario presenta resultados globales buenos o excelentes, lo que permite continuar con el proceso de validación.

AUTOR/ES: Fernandez Marmaneu, A ${ }^{1}$, Cervera Gasch, A. ${ }^{1}$, Gonzalez Chorda VM ${ }^{1}$., Macia Soler L ${ }^{2}$., Salas Medina

$\mathrm{P}^{1}$., Menal Tudela $\mathrm{D}^{1}$.

1. Universitat Jaume I

2. Universidad de Alicante

CORREO ELECTRONICO: cerveraa@uji.es

TELEFONO:

CENTRO DE TRABAJO: Universitat Jaume I, Departamento de Enfermería.

TIPO DE COMUNICACIÓN: Póster $X \quad C \quad$ Comunicación oral

PARA DEFENSA PRESENCIAL: __ _ - PARA DEFENSA VÍA STREAMING: 